\title{
Remote Sensing Methods \\ for Assessing Degradation \\ of Armenia's Highland Pastures
}

\author{
Garegin O. Tepanosyan, Shushanik G. Asmaryan, \\ Vahagn S. Muradyan and Armen K. Sagatelyan* \\ Center for Ecological-Noosphere Studies NAS RA \\ 68 Abovyan Str., Yerevan, 0025, Armenia
}

Received 10.02.2017, received in revised form 20.05.2017, accepted 18.07.2017

In Armenia soil degradation is determined by different factors, including overgrazing, and is a grave concern in terms of food safety and sustainable development. Assessing soil degradation is essential to reveal probable consequences and potential management measures. This article considers a possibility of determining degradation related soil surface components (fractional vegetation cover $-F V C$, bare soils fractions $-B S F$ and surface rock cover - SRC) with help of linear spectral unmixing (LSU) and NDVI-SMA methods, using a QuickBird satellite imagery, and their applicability to assessment and mapping of degradation degree of pasturelands. The results have indicated that LSU and NDVI-SMA methods as applied to a QuickBird satellite image gives a unique opportunity to precisely determine FVC and BSF, whereas the proposed soil degradation assessment and mapping method adequately reflects the actual situation.

Keywords: soil degradation, erosion, overgrazing, remote sensing, linear spetral unmixing, QuickBird imagery.

Citation: Tepanosyan G.O., Asmaryan Sh.G., Muradyan V.S., Sagatelyan A.K. Remote sensing methods for assessing degradation of armenia's highland pastures, J. Sib. Fed. Univ. Eng. technol., 2017, 10(6), 764-774. DOI: 10.17516/1999-494X2017-10-6-764-774.

(C) Siberian Federal University. All rights reserved

* Corresponding author E-mail address: garik.tepanosyan@cens.am 


\title{
Дистанционные методы оценки
}

\section{деградации высокогорных пастбищ Армении}

\author{
Г.О. Тепаносян, Ш.Г. Асмарян, \\ В.С. Мурадян, А.К. Сагателян \\ Центр эколого-ноосферных исследований НАН РА \\ Армения, 0025, Ереван, ул. Абовяна, 68
}

\begin{abstract}
В Армении деградачия почв обусловлена разными факторами, в том числе и чрезмерным стравливанием пастбищ, и является серьезной проблемой с точки зрения продовольственной безопасности и устойчивого развития. Оченка деградации почв важна для определения возможных последствий и потенциальных мер управления. В данной работе рассматривается возможность определения отдельных компонентов земной поверхности, связанных с деградаџией (ППР, ППОП и ППК), методами ЛСР и НРВИ-АСС, используя космический снимок QuickBird, и их применимость для оценки и картографирования деградированности пастбищных земель. Как показывают результаты, методы ЛСР и НРВИ-АСС применительно к космическому снимку QuickBird дают уникальную возможность для точного определения ППР, ППОП, а предложенный метод оценки и картографирования деградацчи почв адекватно отражает реальную ситуацию.
\end{abstract}

Ключевые слова: деградация почв, эрозия, стравливание пастбищ,, дистанцฺионноезондирование, линейное спектральное разделение, снимки QuickBird.

\section{Введение}

Бессистемное, нерациональное, научно не обоснованное использование горных пастбищ приводит к их деградации, что в свою очередь вызывает нарушение природного равновесия и снижение продуктивности сельскохозяйственых земель, становясь серьезной проблемой с точки зрения продовольственной безопасности и устойчивого развития в целом [1-3].

Армения - типичная горная малоземельная страна, где деградация почв, обусловленная бессистемным и черезмерным выпасом скота, очень распространена, особенно на пастбищах, расположенных вблизи деревень, и это в том случае, когда удаленные пастбища используются ограниченно $[4,5]$.

Оценка деградации почв важна для определения возможных последствий и потенциальных мер управления. Из сушествуюших методов оценки деградации методы дистанционного зондирования (ДЗ) и геоинформационные технологии (ГИС) в последние десятилетия стали необходимостью для мониторинга и оценки деградации пастбищ [6-8]. Особый интерес существует к методу линейного спектрального разделения (ЛСР), который имеет большой потенциал выявления и картографирования деградированных земельных участков. Оно широко используется для определения компонентов земной поверхности, связанных с деградацией: проективное покрытие рестительности, оголенных почв и камней (ППР, ППОП, ППК) [9-14].

В методе ЛСР предполагается, что спектральная характеристика данного пикселья является линейной комбинацей эндмемберов (endmember). Эндмемберы - это чистые компоненты земной поверхности, которые, как предполагается, имеют уникальные спектральные характе-

$$
-765-
$$


ристики $[9,15,16]$. В анализе спектральных смесей (ACC) первый и самый важный шаг - это определение эндмемберов. Из существуюших многочисленных методов [17-20] самым распостраненный, вероятно, метод, предложенный Боардманом, который включен в программное обеспечение ENVI (Environment for Visualizing Images) [21].

Сильно упрощенной моделью АСС является модель НРВИ-АСС, основанная на НРВИ (нормализованный разностный вегетационный индекс-NDVI), в котором предпологается, что данный пиксель состоит только из двух эндмемберов - растительность и оголенные почвы, следовательно, значение НРВИ этого пикселя есть линейная комбинация этих двух компонентов. Модель НРВИ-АСС широко используется для определения растительного покрова [15, 22], который признан самым распостраненным индикатором оценки деградации пастбищ методами ДЗ [23-26].

Целью данного исследования является оценка точности методов ЛСР и НРВИ-АСС для определенния отдельных компонентов земной поверхности, связанных с деградацией, используя космический снимок QuickBird, и определение их применимости для картографирования деградации горных пастбищ.

\section{1. Обьект и информационное обеспечение исследования}

Объектами исследования выступают пастбища, расположенные вблизи сельской общины Неркин Сасунашен, Армения (405'ㄹ, $40^{\circ} 21^{\prime} \mathrm{N}$ ) (в целом $\approx 416$ га) (рис. 1). Територия характеризуется сильной каменистостью почв. Норма осадков в период с июля по август составляет 50-150 мм, средняя температура воздуха в июле варьирует в пределах 10-20 ${ }^{\circ} \mathrm{C}$. Исследуемая территория 5-6 месяцев в году (ноябрь, декабрь - март, май) покрыта устойчивым снежным покровом [27].

Стандартный снимок QuickBird, предоставленный фондом “DigitalGlobe Foundation”, был сделан 3 июля 2010 г. при ясном небе. Геометрическая коррекция снимка сделана самим поставшиком снимков со средней квадратической ошибкой (СКО) - 14 м. Стандартный снимок

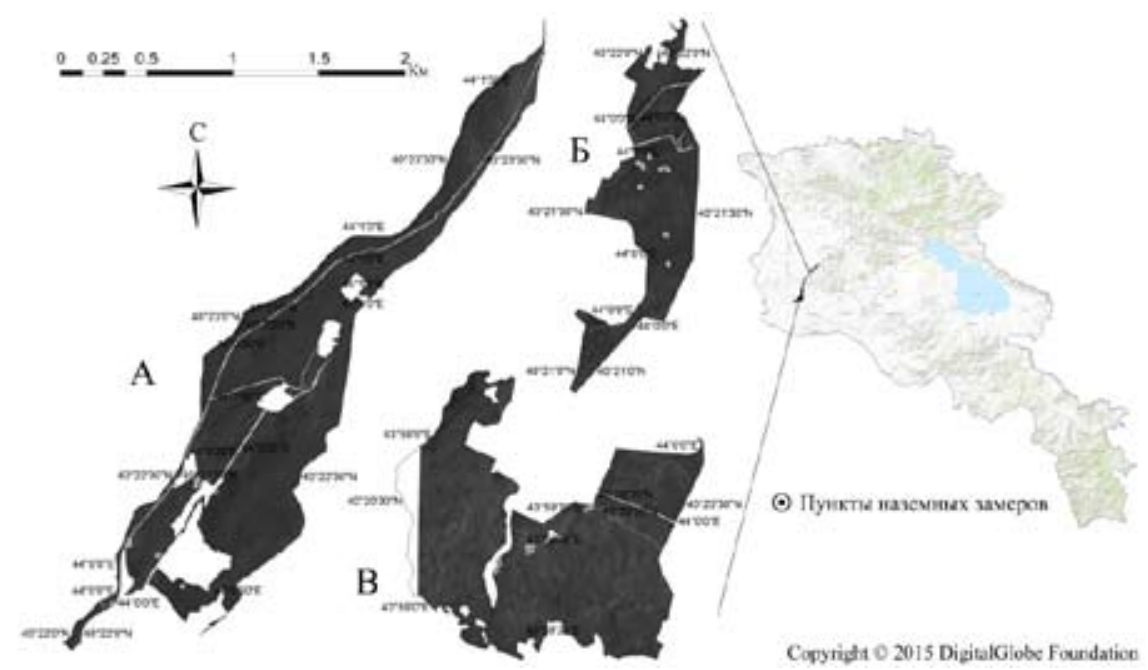

Рис. 1. Расположение исследуемых территорий

Fig. 1. The location of the studied area 
радиометрически скорректирован, а поскольку к нему прилагалась грубая цифровая модель рельефа, ортокоррекция здесь неприемлема. Коррекция включает только специфические ошибки, вызванные инструментом (сенсором), следовательно, необходима дальнейшая коррекция изображения, что и было выполнено согласно техническому руководству DigitalGlobe [28].

Во время полевых работ по визуальным показателям деградации почв (растительный покров, оголенные почвы, обнаженные камни, уплотненные участки почв и распыленность верхнего слоя почвы) $[6,10,13,14,29]$ были выявлены три степени деградированности почв: I - слабо или недеградированные, II - среднедеградированные, III - сильнодеградированные.

На участках с разной степенью деградированности сетка размером $1 \mathrm{~m}^{2}$ (размер клеток $100 \mathrm{~cm}^{2}$ ) была установлена на поверхность земли и вертикально сфотографирована для дальнейшего вычисления в ней ППР, ППОП и ППК в камеральных условиях.

\section{2. Методы}

\section{1. Линейное спектральное разделение и модель НРВИ-АСС}

Математическая формула модели ЛСР представляется следующим образом:

$$
R_{k}=\sum_{i=1}^{M} f_{i} r_{i, k}+e_{k}, \quad \sum_{i=1}^{M} f_{i}=1,0 \leq f_{i} \leq 1,
$$

где $R_{k}$ - величина отражения в каждом спектральном диапазоне $(k)$; $M$ - количество эндмемберов; $f_{i}$ - доля эндмембера $i$ в спектральном диапазоне $k ; r_{i, k}$ - коэффициент отражения эндмембера $i$ в спектральном диапазоне $k$; $e_{k}$ - остаточный член в спектральном диапазоне $k[10,15]$.

В методе ЛСР существуют два вида ограничений. Первое - сумма эндмемберов должна быть равна 1, второе - значения эндмемберов должны находиться в области $0-1[9,15,16]$.

Модель НРВИ-АСС представляет собой простое уравнение:

$$
\text { НРВИ }=f * \mathrm{НРBИ}_{\mathrm{p}}+(1-f) * \mathrm{НРВИ}_{\Pi} \Rightarrow f=\frac{\mathrm{НРВИ}-\mathrm{HРВИ}_{\Pi}}{\mathrm{HРBИ}_{\mathrm{p}}-\mathrm{HРВИ}_{\Pi}}
$$

где $f$ - это доля растительного покрова в смешанном пикселе (т.е. ППР); НРВИ - это НРВИ смешанного пикселя; НРВИ

Для каждого из А, Б и Г участков были выбраны несколько пикселей со $100 \%$-м растительным покровом и 100\%-ми оголенными почвами и рассчитаны их средние значения, что и было принято в качестве НРВИ и НРВЙ . Вставляя полученные результаты (соответственно А- $0.723,0.173$; Б- $0.715,0.186 ;$ Г- $0.7,0.145)$ в уравнение (2), мы получаем значения ППР для каждого участка.

\section{2. Выбор эндмемберов}

Количество эндмемберов зависит от размерности космического снимка [15]. В нашем случае коэффициент корреляции между тремя видимыми спектральными диапазонами снимка составляет 0.98. Это свидетельствует от том, что размерность снимка не столь высока, как предлагается спектральным разрешением. Следовательно, учитывая этот факт и то, что число эндмемберов не должно превышать число спектральных диапазонов снимка, можно предположить, что оптимальное число эндмемберов для нашего исследования - три.

$$
-767-
$$




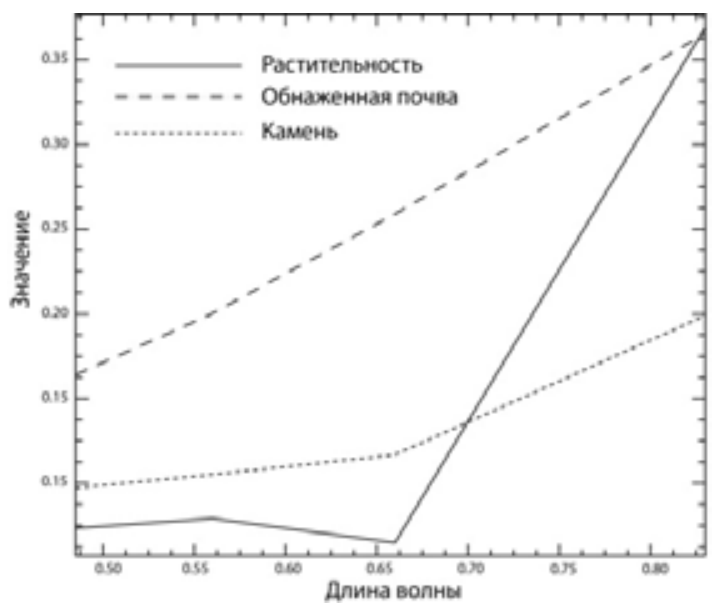

Рис. 2. Кривые усредненных спектральных значений выбранных эндмемберов

Fig. 2. Curves of the averaged spectral values of the selected endmembers

Выбор эндмемберов сделан непосредственно из космического снимка в три этапа. Сперва снимок подвергся обработке с использованием алгоритма спектральной трнсформации (минимизации) шумовых помех (MNF-Minimum noise fraction) [19, 31], потом был применен алгоритм индекса спектральной чистоты пикселя (PPI-Pixel purity index) [32, 33], в результате чего были найдены «самые чистые» пиксели (потенциальные эндмеберы). На последнем этапе, сравнивая спектральные кривые потенциальных эндмеберов со спектральными кривыми спектральных библиотек, а также анализируя панхроматический снимок QuickBird, снимки GoogleEarth высокого разрешения и данные полевых исседований, были выбраны реальные эндмемберы. Таким образом, для каждого из участков А, Б и Г были выбраны три эндмембера - растительность, оголенная почва и камни, средние спектральные значения которых представлены на рис. 2.

\section{3. Результаты и обсуждения}

Карты ППР, ППОП, ППК и СКО, полученные из космического снимка QuickBird методами ЛСР и НРВИ-АСС, изображены на рис. 3. Пиксели со светлой окраской соответствуют высоким значениям эндмеберов, а пиксели с темной - низким значениям. Как видим, вместе с уменьшением высотности наблюдается сокращение растительного покрова, что обусловлено физико-географическими особенностями исследуемой территории, т.е., сменой ландшафтных поясов (от сухостепного до горно-степного поясов).

Согласно низким значениям СКО, полученным для участков А, Б и Г (соответственно $0.000145,0.00077$ l 0.000491), можно предположить, что были отобраны правильные эндмемберы. Однако низкие значения СКО еще не являются гарантией точности полученных данных. Для определения точности нужно сопоставить полученные данные с результатами полевых исследований [10].

На рис. 4 изображены графики линейной регрессии между ППР, ППОП, ППК, полученные методом ДЗ и наземными данными. Результаты показывают существенную корреляцию с коэффициентами детерминации $\left(\mathrm{R}^{2}\right) 0.625$ (метод ЛСР) и 0.636 (метод НРВИ-АСС) для ППР 


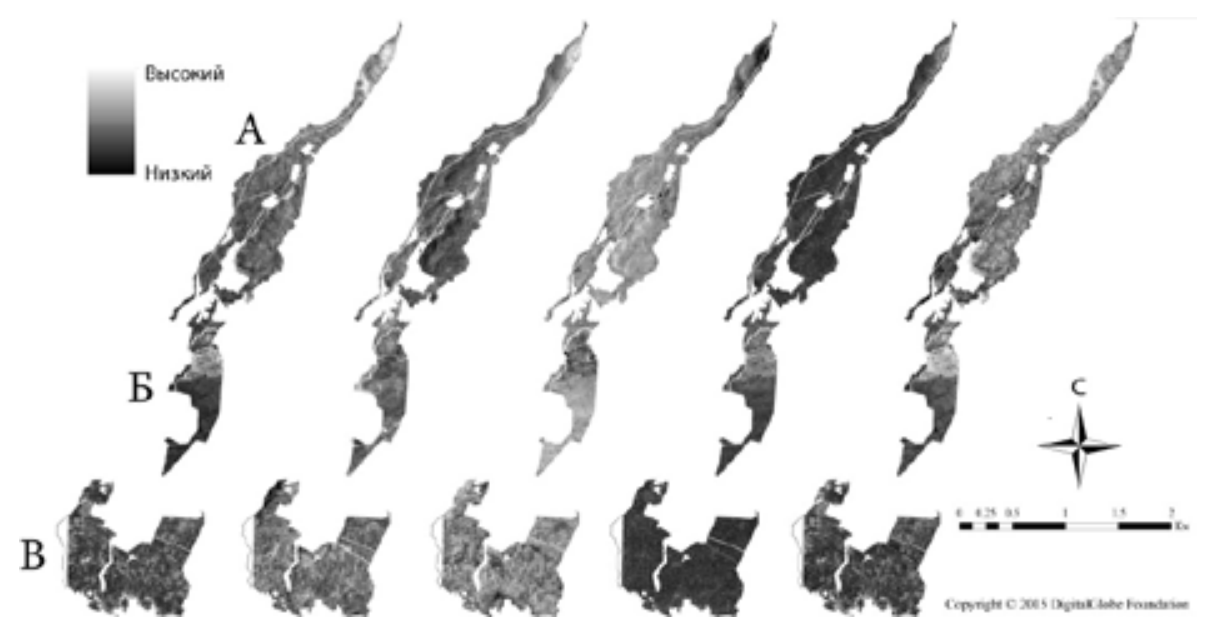

Рис. 3. Карты ППР, ППОП, ППК и СКО, полученные методом ЛСР, и ППР, полученный методом НРВИАСС (слева на право)

Fig. 3. The map of the projective cover of vegetation, bare soil and stones and RMSE obtained by the linear spectral splitting and projective coverage of vegetation obtained by the NDVI-ASM (left to right)
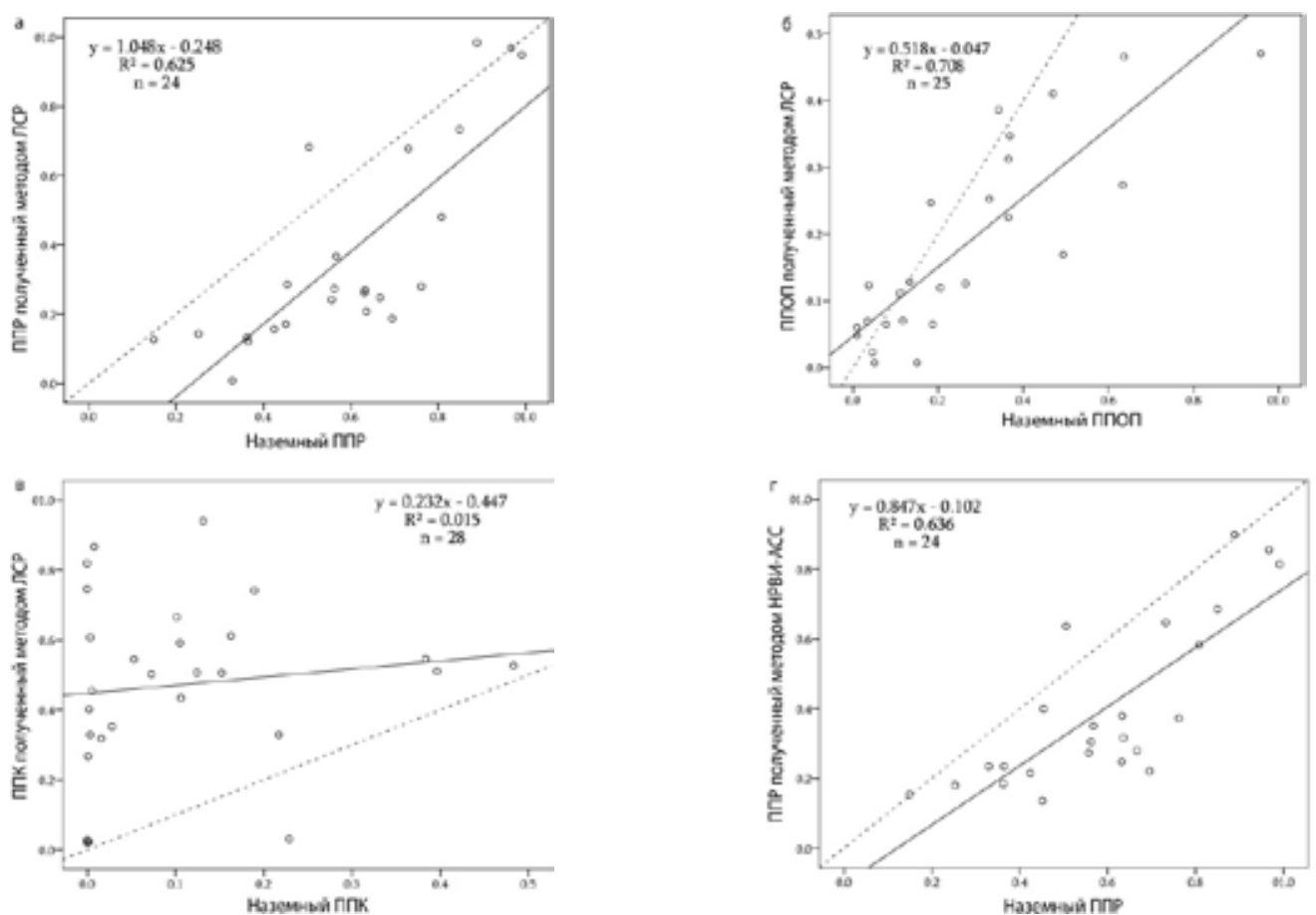

Рис. 4. Графики линейной регрессии между (а) ППР, (б) ППОП, (в) ППК, полученные методом ЛСР, (г) ППР, полученный методом НРВИ-АСС и наземными данными

Fig. 4. The graphs of a linear regression between the projective cover of (a) vegetation, (б) bare soil, (B) stones obtained by the linear spectral splitting, and (г) of projective cover of vegetation obtained by the NDVI-ASM and the ground-based data 


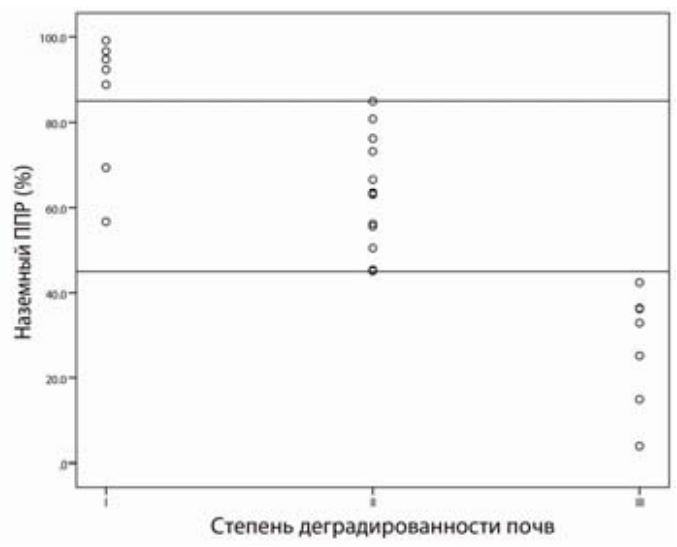

Рис. 5. График сопоставления степени деградированости с ППР

Fig. 5. A graph of the degree of degradation with the projective cover of vegetation

и 0.708 для ППОП (уровень значимости: $\mathrm{p} \leq 0.01)$. Низкое значения $\mathrm{R}^{2}$ для ППК (0.015) означает, что полученные данные не отражают реальные значения ППК в природе.

Используя уравнения регрессии ППР (метод НРВИ-АСС) и ППОП были преобразованы в реальные значения ППР и ППОП.

На рис. 5 видно, что каждой степени деградированности почв соответствует определенный интервал ППР (І степень 85-100 \%, II степень 45-85 \% и III степень 0-45 \%). Видно также, что две точки I степени деградированности не соответствуют общей закономерности в связи с тем, что на участках, соответствующих этим точкам, деградация обусловлена природным фактором - высокой степенью каменистости.

Таким образом, классифицируя ППР по вышеизложенной градации, мы получим карту деградированности пастбищных земел, где, однако, деградация обусловлена как антропогенными, так и природными факторами (каменистость). Для картографирования антропогенной деградации пастбищных земель необходимо отделить деградации, обусловленные природными и антропогенными факторами. В ходе полевых исследований было выяснено, что на участках, где деградация не обусловлена антропогенными факторами, ППОП не превышает 15 \%. Следовательно, путем классификации ППОП по двум классам (<15 \% и 15-100 \%), наложения с уже классифицированной картой ППР и переклассификации мы получим карту андропогенной деградированности почв (рис. 6).

Как видно, около 87 \% территории участка $\boldsymbol{A}$ занимают земли с I степенью деградированности. 11,94 и 0,67 \% всей территории данного участка - это территории соответственно с II и III степенью деградированности. Деградация в основном обусловлена наличием грунтовых дорог, чрезмерным выпасом и вытаптыванием земли, особенно в местах скопления скота (загоны и водопои). На участке $\boldsymbol{\boldsymbol { E }}$ преобладают територии с I и II степенью деградированности, соответственно 47,58 и 47,2 \%. Территории с III степенью деградированности занимают 5,22 \%. На участке $\boldsymbol{B}$ преобладают территории II степени деградированности, а участки с I и III степенью - соответственно 33,12, 14,73 \% всей территории.

В литературе коэффициент детерминации линейной регрессии между ППР, полученной методами ЛЦР, НРВИ-АСС и наземными данными варьирует в пределах $0.85 \approx 0.9[15,25,30,34$, 


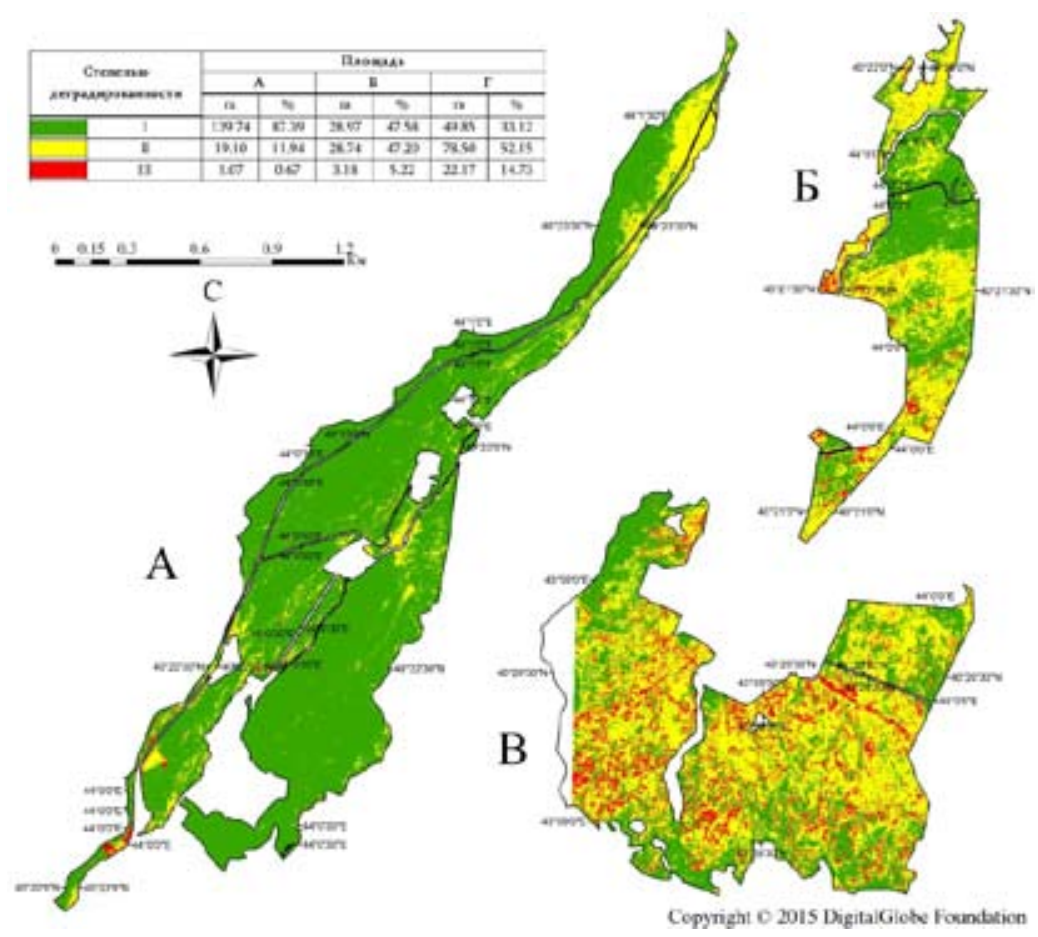

Рис. 6. Карта деградированности сельскохозяйственных земель

Fig 6. The map of the agricultural land degradation

35]. В нашем исследовании зарегистрировано более слабая корреляция $\left(\mathrm{R}^{2}=0.625\right.$ (метод ЛСР) и $\mathrm{R}^{2}=0.636$ (метод НРВИ-АСС)), что в основном может быть связано с временной разницей между спутниковой съемкой и полевыми работами. Для ППОП в сравнении с другими работами были получены достаточно хорошие результаты [10, 25].

Необходимо отметить, что QuickBird не является оптимальным сенсором для определения ППК методом ЛСР. К примеру, в космическом снимке QuickBird отсутствует коротковолновый инфракрасный спектральный диапазон, который очень важен для определения ППК [25]. Для получения точных значений ППК нужно использовать космические снимки с более высоким спектральным разрешением, например WorldView 2 [14].

Визуальная оценка деградированности почв с помощью панхроматического снимка QuickBird показала четкое соответствие с полученной картой эродированности. Это свидетельствует о том, что предложенный метод обьективно отражает реальную ситуацию.

\section{Заключение}

Методы ACC применительно к космическим снимкам QuickBird предоставляют возможность для более точной оценки состояния почв с точки зрения деградации. Это исследование показало, что при помощи АСС можно получить значения ППР и ППОП с достаточной точностью, чтобы использовать их для оценки и картографирования деградации земель. Тем не менее QuickBird из-за низкого спектрального разрешения не является оптимальным сенсором для получения ППК. Следует отметить, что в дальнейшем полученные результаты могут использоваться как входные данные для моделей оценки риска эрозии почв. 
Предложенный метод оценки и картографирования деградации почв может служить альтернативой сложным моделям оценки деградации, потому что большое количество необходимых входных данных для этих моделей в наличии нет, а получение новых данных занимает много времени и требует больших финансовых затрат.

\section{Список литературы}

[1] Zhihui G., Peijun S., Jin C. Estimation of grassland degradation based on historical maximum growth model using with remote sensing data. The International Archives of the Photogrammetry, Remote Sensing and Spatial Information Sciences, 2008, 37(B8), 895-900.

[2] Добровольский Г.В. Деградация и охрана почв. М.: Изд. Московского университета, 2002, 651 c. [Dobrovolskiy G.V. Soil degradation and protection. M.: Publishing house MSU, 2002, 651 p. (in Russian)]

[3] Pimental D. Soil erosion: A food and environmental threat. Environment, Development and Sustainability. Springer, 2006, 8(1), 119-137.

[4] Aleksanyan K., Muradyan V. Geoecological consequences of degradation of Armenia's soil. In: L. Valesyan, ed. Geographical Science in Armenia: the Present and Future, 2006 Yerevan: Publ.h. YSU, 385-392.

[5] Saghatelyan A., Asmaryan Sh., Muradyan V., Tepanosyan G. The Utility for Assessing the Ecological State and Managing Armenian's Farmlands. Journal of Geological Resource and Engineering, 2014, 4, 222-228.

[6] Ballayan D. Soil degradation: Biodiversity and land degradation. ESCAP environment statistics course, FAO, 2000, Rome.

[7] Jafari R., Lewis M.M., Ostendorf B. An image-based diversity index for assessing land degradation in an arid environment in South Australia. Journal of Arid Environment, 2008, 72, 1282 1293.

[8] Qingzhu G., Yu'e L., Yunfan W., Erda L., Wei X., Wangzha J., Baoshan W., Wenfu L. Grassland degradation in Northern Tibet based on remote sensing data. J. Geographical Sciences, 2006, 16(2), 165-173.

[9] De Asis A.M., Omasa K. Estimation of vegetation parameter for modeling soil erosion using linear Spectral Mixture Analysis of Landsat ETM data. ISPRS Journal of Photogrammetry \& Remote Sensing, 2007, 62, 309-324.

[10] De Asis A.M., Omasa K., Oki K., Shimizu Y. Accuracy and applicability of linear spectral unmixing in delineating potential erosion areas in tropical watersheds. International Journal of Remote Sensing, 2008, 29(14), 4151-4171.

[11] Haboudane D., Bonn F., Royer A., Sommer S., Mehl W. Land degradation and erosion risk mapping by fusion of spectrally-based information and digital geomorphometric attributes. International Journal of Remote Sensing, 2002, 23(18), 3795-3820.

[12] Koch M. Geological controls of land degradation as detected by remote sensing: a case study in Los Monegros, north-east Spain. International Journal of Remote Sensing, 2000, 21(3), 457-473.

[13] Metternicht G.I., Fermont A. Estimating Erosion Surface Features by Linear Mixture Modeling. Remote Sensing of Environment, 1998, 64(3), 254-265. 
[14] Lehnert L.W., Meyer H., Meyer N., Reudenbach Ch., Bendix J. A hyperspectral indicator system for rangeland degradation on theTibetan Plateau: A case study towards spaceborne monitoring. Ecological Indicators, 2014, 39, 54-64.

[15] Xiao J., Moody A. A. comparison of methods for estimating fractional green vegetation cover within a desert-to-upland transition zone in central New Mexico, USA. Remote Sensing of Environment, 2005, 98, 237-250

[16] Plaza A., Martínez P., Pérez R., Plaza J. Spatial/Spectral Endmember Extraction by Multidimensional Morphological Operations. IEEE Transaction on Geoscience and Remote Sensing, 2002, 40(9), 2025-2041.

[17] Bioucas-Dias J.M., Nascimento J. Hyperspectral subspace identification. IEEE Transactions of Geoscience and Remote Sensing, 2005, 46 (8), 2435-2445.

[18] Camps-Valls G., Tuia D., Gómez-Chova L., Jiménez S., Malo J. Remote Sensing Image Processing. Morgan \& Claypool, 2012, 176 p.

[19] Green A.A., Berman M., Switzer P., and Craig M.D. A transformation for ordering multispectral data in terms of image quality with implications for noise removal. IEEE Transactions of Geoscience and Remote Sensing, 1988, 26 (1), 65-74.

[20] Lee J.B., Woodyatt A.S., Berman M. Enhancement of high spectral resolution remotesensing data by noise-adjusted principal components transform. IEEE Transactions of Geoscience and Remote Sensing, 1990, 28 (3), 295-304.

[21] Berman M., Kiiveri H., Lagerstrom R., Ernst A., Dunne R., Huntington J. F. ICE: A Statistical Approach to Identifying Endmembers in Hyperspectral Images. IEEE Transaction on Geoscience and Remote Sensing, 2004, 42(10), 2085-2095.

[22] Gutman G., Ignatov A. The derivation of the green vegetation fraction from NOAA/AVHRR data for use in numerical weather prediction models. International Journal of Remote Sensing, 1998, 19 (8), 1533-1543.

[23] Akiyama T., Kawamura K. Grassland degradation in China: methods of mon-itoring, management and restoration. Grassland science, 2007, 53(1), 1-17.

[24] Gao Q.Z., Wan Y.F., Xu H.M., Li Y., Jiangcun W.Z., Borjigidai A. Alpine grass-land degradation index and its response to recent climate variability in NorthernTibet, China. Quaternary International, 2010, 226(1-2), 143-150.

[25] Meusburger K., Banninger D., Alewell C. Estimating vegetation parameter for soil erosion assessment in an alpine catchment by means of QuickBird imagery. International Journal of Applied Earth Observation and Geoinformation, 2010, 12, 201-207.

[26] Pickup G., Bastin G.N., Chewings V.H. Remote-sensing-based condition assessment for nonequilibrium rangelands under large-scale commercial graz-ing. Ecological Applications, 1994, 4(3), 497-517.

[27] Погосян Д.А. Сельскохозяйственная оценка природных ресурсов территории Армянской ССР. Издательство АН Армянской ССР. Ереван, 1986, 221 с.

[28] Krause K. Radiometric Use of QuickBird Imagery, Technical Note. DigitalGlobe, Longmont, Colorado, USA, 2005, 17 p.

[29] Stocking M., Murnaghan N. Handbook for the Field Assessment of Land Degradation. Earthscan Publications Ltd., 2000, 184 p.

$$
-773-
$$


[30] Verger A., Martinez B., Camacho-de Coca F., Garcia-Haro F.J. Accuracy assessment of fraction of vegetation cover and leaf area index estimates from pragmatic methods in a cropland area. International Journal of Remote Sensing, 2009, 30(10), 2685-2704.

[31] Шовенгердт Р.А. Дистанционное зондирование. Методы и модели обработки изображений. М.: Техносфера, 2010, 560 с.

[32] Boardman J.W. Automated spectral unmixing of AVIRIS data using convex geometry concepts. In: Summaries, Fourth JPL Airborne Geoscience Workshop, JPL Publication 93-26, 1993, $11-14$.

[33] Boardman J.W., Kruse F.A., Green R.O. Mapping target signatures via partial unmixing of AVIRIS data. In: Summaries, Fifth JPL Airborne Geoscience Workshop, JPL Publication 95-1, 1995, 23-26.

[34] Elmore A.J., Mustard J.F., Manning S.J., Lobell D.B. Quantifying Vegetation Change in Semiarid Environments Precision and Accuracy of Spectral Mixture Analysis and the Normalized Difference Vegetation Index. Remote Sensing of Environment, 2000, 73(1), 87-102.

[35] Zhang X., Liao Ch., Li J., Sun Q. Fractional vegetation cover estimation in arid and semiarid environments using HJ-1 satellite hyperspectral data. International Journal of Applied Earth Observation and Geoinformation, 2013, 21, 506-512. 